\title{
STRONG POLARIZATION SHOCKS IN THE INTERSTELLAR SPACE
}

\author{
V.C. LIU and D. BASTOS-NETTO \\ The University of Michigan, Ann Arbor, Michigan 48104, USA
}

Received 21 March 1972

\begin{abstract}
Structure of infinitely strong shocks in a slightly ionized gas, suitable for the interstellar HI region, is discussed. The charge-separation field, in closed form, is obtained by using a bi-modal (beam-continuum) distribution and the Mott-Smith approach.
\end{abstract}

The shellings of envelopes from novae and super novae can occur in interstellar space at extremely high speeds reaching $6000-7000 \mathrm{~km} / \mathrm{sec}$. Such explosive radially-expanding flows of the interstellar gases have often been treated hydrodynamically considering their leading edges as advancing spherical shock fronts $[1,2]$. The consequences of such fast-propagating shock waves have been construed as causes of various astrophysical phenomena, e.g., the origin of cosmic rays among others.

Consider a shock front propagating in the interstellar HI region where the electron density is small compared with the neutral atomic density $\left(n_{\mathrm{e}} \sim 10^{-3} n_{\mathrm{H}}\right)$. The presence of large gradient of electron density in an extreme compression shock and the relatively high mobility of electron gas resulting from their small mass cause the diffusion of the electron gas with respect to the ion gas hence the creation of charge separation and its associated polarization field in the shock transition layer. The presence of this electrical field in the shock front which apparently has been unnoticed so far could be astrophysically important. To focus attention upon this salient feature of the interstellar shocks, a simplified model is constructed to describe the interaction between an advancing one-dimensional shock front and the ambient interstellar medium (HI) of which the magnetic field and relativistic particle effects are neglected. It is further assumed that the degree of ionization of the gas remains constant in the region of interest and the radiation energy loss is still small [1]. A review of the literature shows the lack of adequate discussion on the extremely strong shocks in a slightly ionized gas $[1,2]$. A unique feature of the present shock problem is that $u_{1}$ (shock speed) $\gg v_{\mathrm{e} 2}$ (electron thermal speed behind the shock) hence the low gradient approximation of electron distribution commonly used elsewhere [3] is not suitable here.

Recognizing the fact that the distributions of the particle species (electrons, e; ions, $\mathrm{i}$ and neutrals, $\mathrm{H}$ ) in the upstream, namely where $x \rightarrow-\infty$ considering the shock front fixed on $y, z$-plane, become $\delta$-functions in the velocity spaces and act like atomic beams in the physical space. This family of particles transform, throughout the shock transition layer, by collisions of predominately charged-neutral and the neutral-neutral types in view of the condition: $n_{\mathrm{e}} \approx n_{\mathrm{i}} \leqslant n_{\mathrm{H}}$, into those constituting the hot continuum flow downstream. In other words the region of the shock "discontinuous" change can be regarded as a region of beam-convection and decay into a hot bath of minute forward velocity relative to the shock front. It is therefore appropriate to use a bi-modal approximation of the steady particle distributions: $f_{\mathrm{s}}(x, \boldsymbol{c})=F_{\delta \mathrm{s}}(x, c)_{h}+F_{\mathrm{s}}(x, c)$ where the beam component $F_{\delta \mathrm{s}}=N_{\mathrm{s} 1}(x) \delta\left(c-u_{1}\right)$ and the continuum component $F_{\mathrm{s}}=N_{\mathrm{s}}(x)\left(\beta_{\mathrm{s}} / \pi\right)^{3 / 2} \exp -\beta_{\mathrm{s}}\left[c-u_{\mathrm{s}}(x)\right]^{2}$ with the usual boundary conditions: $N_{\delta \mathrm{s}}(-\infty)=n_{\mathrm{s} 1}$ (ambient number densities), $N_{\delta \mathrm{s}}(+\infty)=0$ and $N_{\mathrm{s}}(-\infty)=0$, $N_{\mathrm{s}}(+\infty)=n_{\mathrm{s} 2}$ (number densities behind the shock) where $\beta_{\mathrm{s}}=m_{\mathrm{s}} / 2 K T_{\mathrm{s}}(x)$ and subscript s stands for electrons, e; ions, i or neutrals, H. Notice that $F_{\mathrm{s}}$ is a locally Maxwellian distribution [5].

The Mott-Smith procedure [4] generalized for slightly ionized gases will be followed to obtain a solvable system of differential equations for the $x$-dependent functions $N_{\mathrm{s} 1}, N_{\mathrm{s}}, T_{\mathrm{s}}$, and $u_{\mathrm{s}}$ by substituting $f_{x}(x, c)$ into the Boltzmann equation [5] retaining only the $(e, \mathrm{H})$-collisions for the $f_{\mathrm{e}}$-equation, the $(\mathrm{i}, \mathrm{H})$-collisions for the 
$f_{i}$-equations and using a fifth power law (Maxwellian) force interaction which is suitable for a charged-neutral coilision. Under these conditions the distribution of the neutral species $(\mathrm{H})$, except for providing collision partners, can be treated independently of the charged species. Such substitutions for the equations of the charged species lead to the component Boltzmann-like equations of electrons $\left(q_{\mathrm{e}}=-e\right)$ and ions $\left(q_{\mathrm{i}}=e\right)$, after the collision term $J\left[F_{\delta \mathrm{s}}+F_{\mathrm{s}}, F_{\delta \mathrm{H}}+F_{\mathrm{H}}\right]$ has been expanded and simplified, as follows (with subscripts denoting the electrons and ions only):

$c_{x} \frac{\partial F_{\delta \mathrm{s}}}{\partial x}+\frac{q_{\mathrm{s}} E(x)}{m_{\mathrm{s}}} \frac{\partial F_{\delta \mathrm{s}}}{\partial c_{x}}+\nu_{\mathrm{H}, \mathrm{s}} F_{\delta \mathrm{s}}=J\left[F_{\delta \mathrm{s}}, F_{\delta \mathrm{H}}\right]$

$c_{x} \frac{\partial F_{\mathrm{s}}}{\partial x}+\frac{q_{\mathrm{s}} E(x)}{m_{\mathrm{s}}} \frac{\partial F_{\mathrm{s}}}{\partial c_{x}}+\nu_{\delta \mathrm{H}, \mathrm{s}} F_{\mathrm{s}}-J_{1}\left[F_{\delta \mathrm{s}}, F_{\mathrm{H}}\right]-J_{1}\left[F_{\mathrm{s}}, F_{\delta \mathrm{H}}\right]=J\left[F_{\mathrm{s}}, F_{\mathrm{H}}\right]$

where the standard notations [5] are used, e.g.,

$J\left[F_{\mathrm{s}}, F_{\mathrm{H}}\right] \equiv \iiint\left(F_{\mathrm{s}}^{\prime} F_{\mathrm{H}}^{\prime}-F_{\mathrm{s}} F_{\mathrm{H}}\right)\left|c_{\mathrm{s}}-c_{\mathrm{H}}\right| b \mathrm{~d} b \mathrm{~d} \epsilon \mathrm{d} c_{\mathrm{H}}$

$J_{1}\left[F_{\mathrm{s}}, F_{\delta \mathrm{H}}\right] \equiv \iiint F_{\mathrm{s}}^{\prime} F_{\delta \mathrm{H}}^{\prime}\left|c_{\mathrm{s}}-c_{\mathrm{H}}\right| b \mathrm{~d} b \mathrm{~d} \epsilon \mathrm{d} c_{\mathrm{H}} ; \quad \nu_{\mathrm{H}, \mathrm{s}} \equiv \iiint F_{\mathrm{H}}\left|c_{\mathrm{s}}-c_{\mathrm{H}}\right| b \mathrm{~d} b \mathrm{~d} \epsilon \mathrm{d} c_{\mathrm{H}}$.

Notice that eqs. (1) and (2) state that beam-beam interactions remain in the beam population, while beamcontinuum interactions move both partners into the continuum population.

The Boltzmann-like equations (1) and (2), subsequent to multiplications successively by $1, c_{x}$ and $c^{2}$ and integrations over velocity spaces give conservation equations of mass, momentum and energy which, together with the charge conservation condition: $\int c f_{\mathrm{i}} \mathrm{d} c=\int c f_{\mathrm{e}} \mathrm{d} c$ and the Poisson equation: $\mathrm{d} E / \mathrm{d} x=$

$4 \pi e\left[\int f_{\mathrm{i}} \mathrm{d} c-\int f_{\mathrm{e}} \mathrm{d} c\right]$, constitute a self-consistent system of equations for the shock structure. After some algebraic simplification of these equations, an equation of polarization field $E(x)$ in the shock layer is obtained as follows:

$E \mathrm{~d} E / \mathrm{d} x=A(x)+B(x) E+C(x) E^{2}$

where

$$
\begin{aligned}
& A(x)=\left(\frac{K T_{2}}{e d_{2}}\right)^{2}\left\{\left(1-4 \frac{N_{\delta}}{N_{2}}\right)\left(\frac{u^{2}}{u_{2}^{2}}-\frac{16}{5} \frac{u}{u_{2}}+4\right)\left(\frac{5}{16} \frac{\mathrm{d}}{\mathrm{d} x} \frac{u}{u_{2}}\right)+\right. \\
& \left.+2\left(\frac{u}{u_{2}}-1\right)\left(\frac{u}{u_{2}}-4\right)\left(1-\frac{5}{8} \frac{u}{u_{2}}\right) \frac{\mathrm{d}}{\mathrm{d} x} \frac{N_{\delta}}{N_{2}}\right) / \frac{15}{8} \frac{u}{u_{2}}\left(1-\frac{5}{8} \frac{u}{u_{2}}\right) \text {. } \\
& B(x)=\frac{8}{5} \pi e n_{\mathrm{e}}\left(1-\frac{5}{8} \frac{u}{u_{2}}\right)\left[\left(1+\exp \frac{x}{l_{\mathrm{HH}_{2}}}\right)^{-l_{\mathrm{HH}_{2}} l_{\mathrm{HH}_{2}}}-\left(1+\exp \frac{x}{l_{\mathrm{HH}_{2}}}\right)^{-l_{\mathrm{eH}_{2}} h_{\mathrm{HH}_{2}}}\right] \text {; } \\
& C(x)=\frac{1}{2}\left(\frac{\mathrm{d}}{\mathrm{d} x} \frac{u}{u_{2}}\right) / \frac{u}{u_{2}}\left(1-\frac{5}{8} \frac{u}{u_{2}}\right) ; \quad N_{\delta}=\sum_{\mathrm{s}} N_{\delta \mathrm{s}} ; \quad N_{2}=\sum_{\mathrm{s}} N_{\mathrm{s} 2}
\end{aligned}
$$

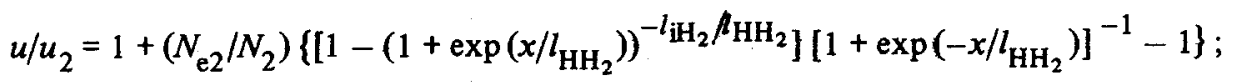

with $d_{2}$ and $l_{\mathrm{sH}}$ denoting the Debye length [5] and the collisional (s, $\left.\mathrm{H}\right)$ mean free path respectively (subscript 2 for states behind the shock). 
Eq. (3) for the case where the mean free paths of electron-neutral and ion-neutral interactions behind the shock are approximately equal, namely $l_{\mathrm{eH}_{2}} \sim l_{\mathrm{iH}_{2}}$, can be solved in closed form [6] :

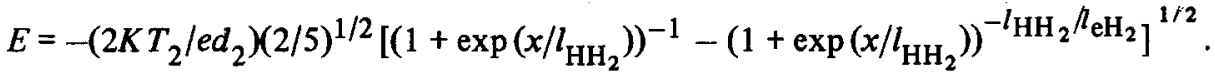

It should be hoted that the condition of frozen ionization herein assumed is expected to hold near the leading edge of the shock wave. As the ionization level rises toward the trailing edge the particle collision effect would be eventually governed by the Fokker-Planck process. In addition the plasma micro-instabilities become more prominent and tend to create turbulent-like process which enhances randomization of particle distributions and considerably reduces the particle mean free path that characterizes the shock thickness. The discussion on the astrophysical significance of the polarization field will be given elsewhere.

\section{References}

[1] L. Woltjer, I.A.U. Symp. No. 39, ed. H.J. Habing (Reidel 1970) 229;

S.A. Kaplan and S.B. Pikelner, Interstellar medium (Harvard, 1970).

[2] Ya.B. Zeldovich and Yu.P. Raizer, Physics of shock waves and high temperature hydrodynamic phenomena II (Academic Press, 1967).

[3] V.C. Liu, Space Sci. Rev. 9 (1969) 423.

[4] D.L. Turcotte and I.M. Scholnick, Phys. Fluids Suppl. I (1969) 79.

[5] S. Chapman and T.G. Cowling, Mathematical theory of nonuniform gases (Cambridge, 1970).

[6] D. Bastos-Netto, Ph.D. Thesis (University of Michigan, 1971), (available from University Microfilm Co., Ann Arbor, Michigan, USA). 